\title{
Echocardiographic pitfalls in the diagnosis of hypertrophic cardiomyopathy
}

\author{
Krishna Prasad, John Atherton, Gillian C Smith, William J McKenna, \\ Michael P Frenneaux, Petros Nihoyannopoulos
}

\section{Department of Cardiology, University of Wales College of Medicine, Heath Park, Cardiff CF4 4XN, UK K Prasad \\ J Atherton \\ M P Frenneaux}

\section{Department of Cardiological Sciences, St George's Hospital Medical School, London SW17 ORE, UK \\ G C Smith \\ W J McKenna}

Imperial College School of Medicine and Technology, National Heart and Lung Institute, Cardiology Department, Hammersmith Hospital, Du Cane Road, London W12 0NN, UK P Nihoyannopoulos

Correspondence to: Dr Nihoyannopoulos
The diagnosis of hypertrophic cardiomyopathy (HCM) can often be difficult. Traditionally, it has been a diagnosis of exclusion, requiring the demonstration of left ventricular hypertrophy $(\mathrm{LVH})$ in the absence of other causes, such as systemic hypertension or aortic stenosis. ${ }^{12}$ Early reports focused on the presence of asymmetrical hypertrophy with an outflow tract gradient (hence the acronym HOCM) but it has become clear that this is not the most common appearance. ${ }^{3-7}$ Furthermore, recent reports of genotypically affected individuals without hypertrophy, who are nevertheless at risk of sudden death, ${ }^{8}$ further complicate the situation. Recently, McKenna and colleagues ${ }^{9}$ proposed modified criteria for the diagnosis of HCM, which overcome some of the problems associated with the conventional criteria (table 1). The new proposed criteria highlight the importance of a comprehensive family history and 12 lead electrocardiogram (ECG) over the echocardiogram in the diagnosis of HCM. Even then, extreme care must be taken because systematic family screening programmes have identified patients who are phenotypically normal but have affected siblings and offspring. These carriers can be missed and their progeny miscoded with less rigorous protocols. Diagnosis can only be $100 \%$ reliable when all the implicated gene loci have been identified. Until then the combination of ECG and echocardiography, in conjunction with other clinical information, remain the most useful tests for the diagnosis of patients with HCM. Here, we discuss some of the pitfalls encountered in the echocardiographic evaluation of HCM.

\section{Echocardiographic features of HCM}

As a rule, patients with HCM have a considerably increased left ventricular wall thickness, an echocardiographic marker of ventricular hypertrophy, with a small non-compliant but apparently well contracting left ventricle. Early $M$ mode echocardiographic studies defined the characteristic features as asymmetrical hypertrophy of the ventricular septum, with or without systolic anterior motion of the mitral valve, and premature closure of the aortic valve (fig 1). An inherent disadvantage of $M$ mode echocardiography is that only a small section of the left ventricle can be examined with a single ultrasound beam, usually passing through the anterior septum and posterior walls. The advent of two dimensional echocardiography has permitted a more complete anatomical description, enabling an appreciation of the wide range of disease morphology and spacial
Table 1 Echocardiographic pitfalls

False positive diagnosis of HCM

Conditions simulating LVH/ASH

Oblique sections of ventricular walls (off axis views)

Moderator band (right ventricular)

Left ventricular false tendons

Sigmoid septum of elderly

Other forms of ventricular hypertrophy

Hypertensive patients with inferior myocardial infarction

Right ventricular hypertrophy

Athlete's heart

Infiltrative disorders

Cardiac amyloidosis

Haemochromatosis

Friedreich's ataxia

Incidental detection of outflow tract gradient

Associated with ventricular hypertrophy

Dialysis patients

False negative diagnosis of $\mathrm{HCM}$

Conditions without ASH

Apical hypertrophy

Localised LVH

Mild concentric LVH

Absence of left ventricular hypertrophy

Children and adolescents

Protein binding $\mathrm{C}$ mutations

Troponin T mutations

End stage HCM with dilated fibrotic LV

ASH, asymmetrical hypertrophy of the ventricular septum; HCM, hypertrophic cardiomyopathy; LV, left ventricle; LVH, left ventricular hypertrophy.

distribution of ventricular hypertrophy. ${ }^{10}$ The earlier belief that asymmetrical hypertrophy of the ventricular septum, systolic anterior motion of the mitral valve, and midsystolic closure of the aortic valve were each diagnostic of HCM were dispelled by the demonstration of each of these abnormalities in a variety of other conditions, including patients with hypertensive heart disease. ${ }^{11}{ }^{12}$

\section{Assessment of ventricular hypertrophy}

Echocardiographic assessment in a patient with HCM requires comprehensive imaging of the left ventricle from several projections, including parasternal long axis, serial short axis views, and imaging from the apical and subcostal windows. The parasternal long axis view is of pivotal importance for orientation and the correct beam alignment along the orthogonal planes. It is imperative that the beam transects the left ventricle perpendicularly, because oblique images will lead to the overestimation of wall thickness and cavity dimensions. If only oblique long axis images are attainable, serial short axis measurements should be treated with caution as far as measurements are concerned. The long axis projection examines the profile of the ventricular septum and left ventricular outflow tract, with good visualisation of the aortic valve and mitral valve, including the subvalvar apparatus. 

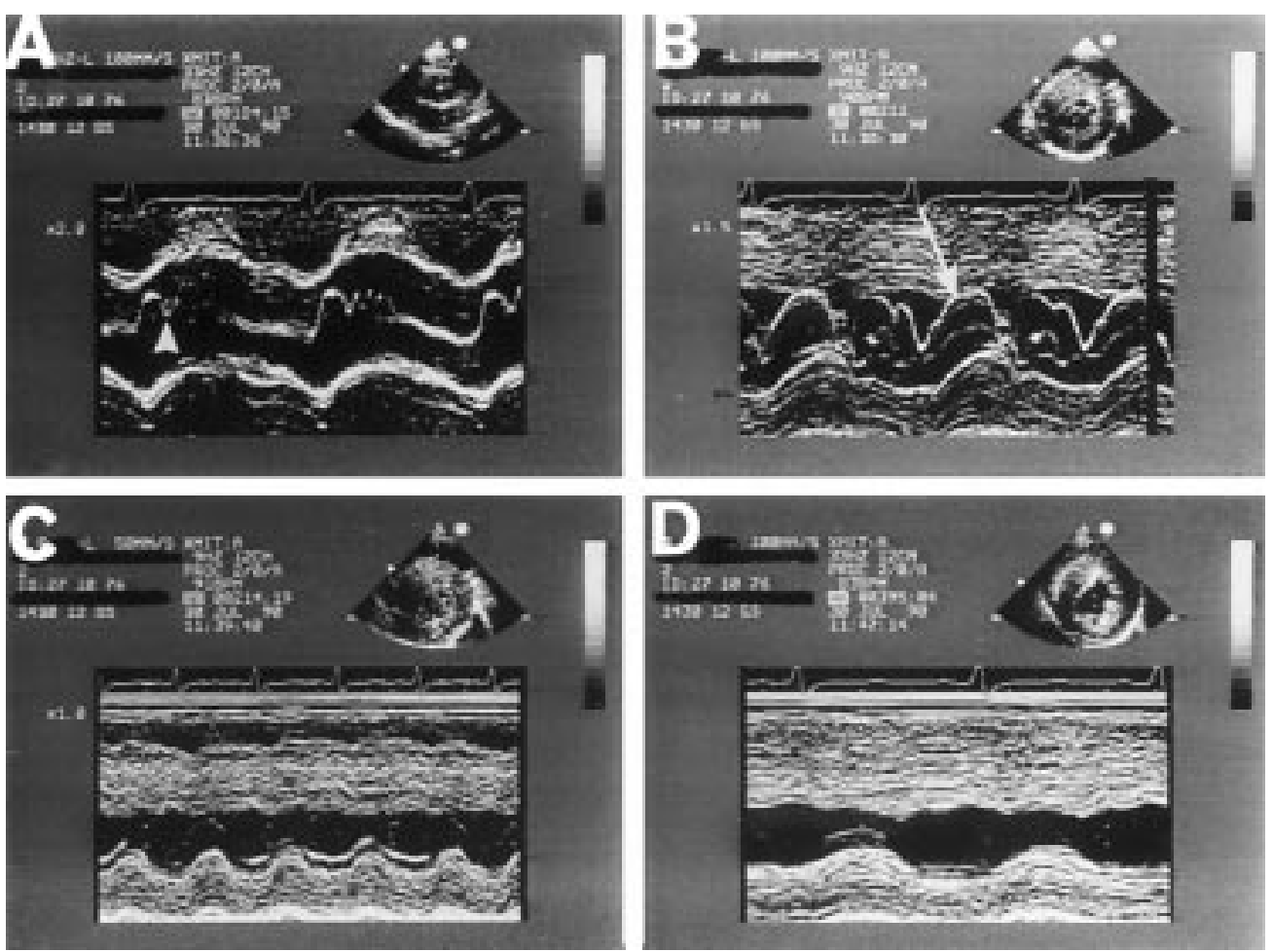

Figure 1 Typical M mode echocardiogram from a patient with hypertrophic cardiomyopathy highlighting the four main echocardiographic features of the condition. (A) Midsystolic closure of the aortic valve (arrowhead); (B) systolic anterior motion of the mitral valve (arrow) and asymmetric left ventricular hypertrophy together with a small, vigorously contracting left ventricle. In frames $(C)$ and $(D)$, the $M$ mode beam passes through the septum and posterior wall beyond the mitral valve, at the level of the papillary muscles and apex, demonstrating the large reduction of left ventricular end systolic dimensions. Reproduced from Nihoyannopoulos and McKenna with permission of Churchill Livingstone. ${ }^{10}$

From this view, the relations between the basal septum, mitral valve, and aortic valve during the cardiac cycle can be appreciated. Increased wall thickness can occur at any site and can be quite localised or eccentric in distribution. ${ }^{7}$ Therefore, it is easy to see how diagnostic features can be missed with a standard $\mathrm{M}$ mode projection or incomplete two dimensional analysis. Multiple short axis sections from the mitral valve level down to the most distal segment, together with good, not foreshortened, apical two and four chamber views, are fundamental to a complete evaluation. Wall thickness should be measured in four

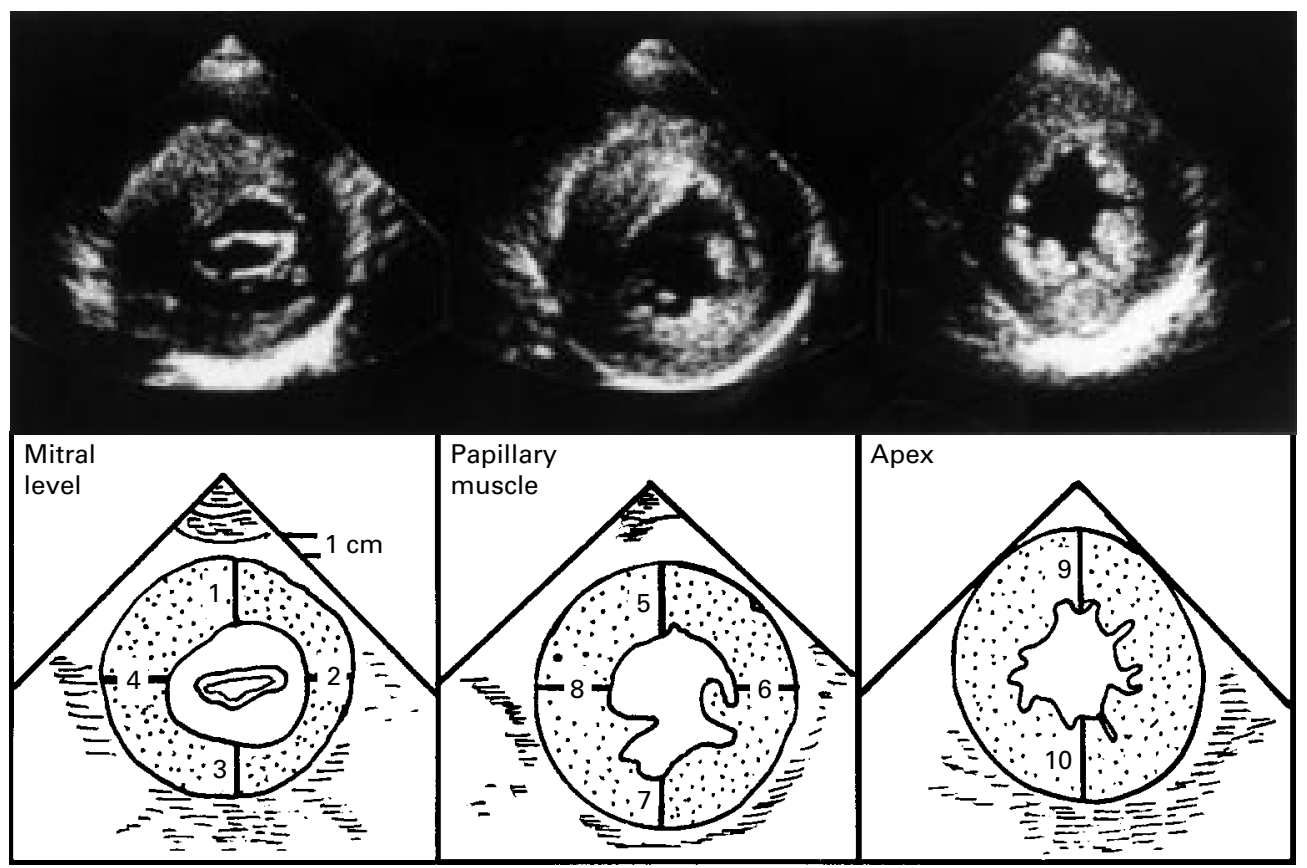

Figure 2 An example of serial short axis, cross sectional views of the left ventricle at three levels - the mitral valve, papillary muscles, and apex - demonstrating the segments of myocardial wall measured routinely in patients with hypertrophic cardiomyopathy in our laboratory. Reproduced from Nihoyannopoulos and McKenna with permission of Churchill Livingstone. ${ }^{10}$ 


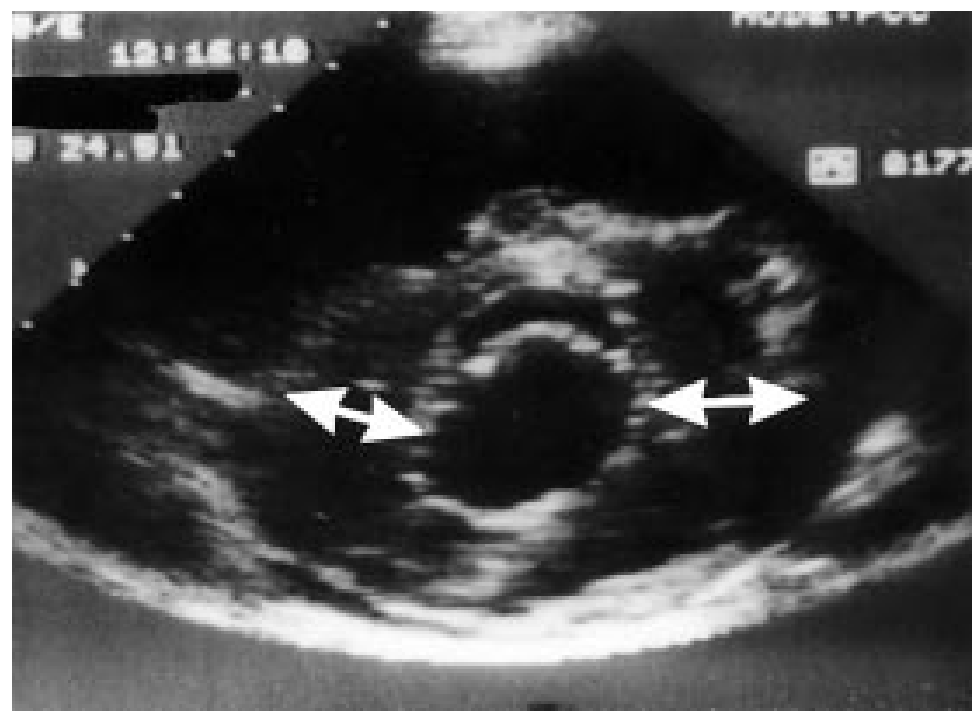

Figure 3 Parasternal short axis view of the left ventricle at the mitral valve level demonstrating a typical eccentric form of ventricular hypertrophy localised essentially at the lateral wall and posterior septum, while the anterior and posterior walls are normal. Reproduced from Nihoyannopoulos and McKenna with permission of Churchill Livingstone. ${ }^{10}$

segments - anterior and posterior septal, lateral and inferior - to characterise the extent and distribution of ventricular hypertrophy (fig 2). Figure 3 provides an example of HCM with an unusual distribution, which could be missed if only measurements in the antero-posterior plane are taken.

Echocardiographically, HCM can be considered as a condition characterised by the following: (1) hypertrophy of all (concentric) or a proportion of the walls of the left ventriclethat is, ventricular septum (asymmetric) or apex (distal); (2) a dilated left atrium; (3) small, non-dilated ventricles; (4) absence of any other cardiac or systemic condition producing hypertrophy (aortic stenosis (such as valvar, subvalvar, supravalvar), coarctation, systemic hypertension, renal failure, or amyloidosis); and (5) normal or supernormal (vigorous) ventricular contraction in the absence of other hyperdynamic states (fever, pregnancy, or

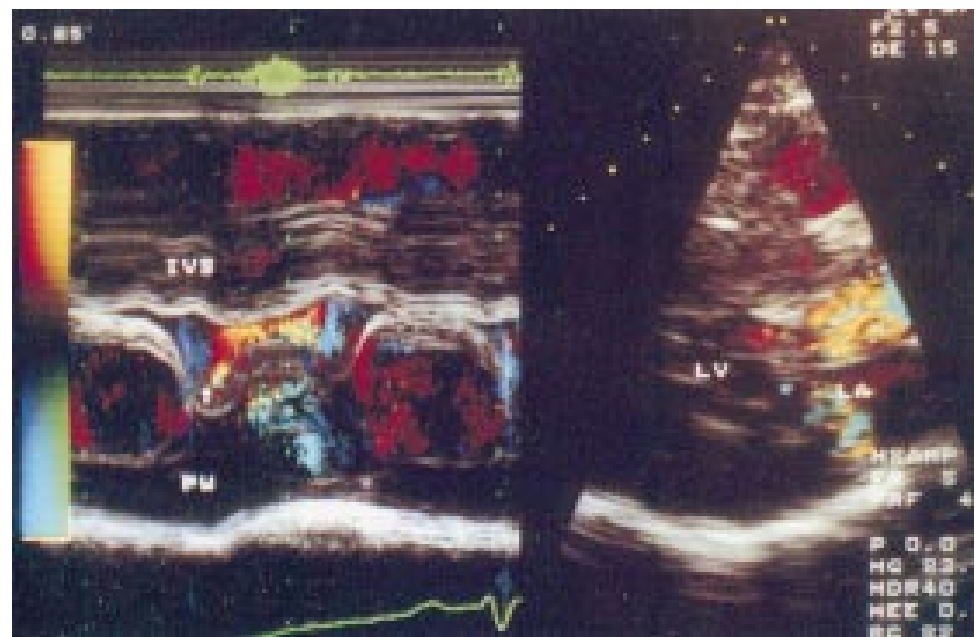

Figure 4 Parasternal long axis view with colour $M$ mode Doppler echocardiography from a patient with hypertrophic cardiomyopathy and a high $(90 \mathrm{mmHg})$ outflow tract gradient. Notice the occurrence of systolic anterior motion of the mitral valve at the same time as the presence of outflow turbulence (arrow), and later into systole, the presence of mitral regurgitation. hyperthyroidism). Associated findings, such as systolic anterior motion of the mitral valve or midsystolic closure of the aortic valve, should not be considered diagnostic on their own. When all the echocardiographic features are present, together with the suggestive clinical picture, a firm diagnosis of HCM can be made. However, when only a small number of these findings is present, the diagnosis can only be made clinically, by exclusion of other causes of ventricular hypertrophy.

\section{Diastolic dysfunction}

The usually small left ventricle in patients with HCM typically exhibits abnormal diastolic function, with evidence of delayed relaxation seen on Doppler echocardiography. ${ }^{10}$ Prolongation of mitral $\mathrm{E}$ wave, abnormal deceleration time, delayed mitral valve opening, prolonged isovolumic relaxation time (IVRT), and abnormal pulmonary venous flow can all describe left ventricular diastolic dysfunction.

Early studies using $M$ mode echocardiography showed that the rates of filling and relaxation of the left ventricle were abnormal in patients with hypertrophic cardiomyopathy. ${ }^{13-15}$ However, although these measurements are sensitive, they are non-specific, time consuming, and require excellent quality recordings, which might only be obtainable in a small number of patients. No single or combination of $M$ mode echocardiographic criteria are diagnostic. ${ }^{16}$ Doppler echocardiographic recordings, on the other hand, are easy to obtain in nearly all patients and provide a good overall estimate of diastolic filling abnormalities of the left ventricle. ${ }^{17-20}$ However, as with $\mathrm{M}$ mode echocardiography, none of the Doppler findings are specific for the diagnosis of HCM, and they should be taken in the overall context of the images. Rarely, HCM patients can exhibit a frankly restrictive filling pattern.

\section{Obstruction in HCM}

The anterior mitral leaflet makes contact with the proximal ventricular septum in about $25 \%$ of patients with HCM. Systolic anterior motion of the mitral valve causes the left ventricular outflow tract to be transiently narrowed during systole, which gives rise to the gradient. This is the usual site of the "obstruction" (fig 4). In about $20 \%$ of patients, systolic anterior motion of the mitral valve might be incomplete-that is, there is anterior displacement of the anterior mitral leaflet but no contact with the septum. However, systolic anterior motion of the mitral valve is a rather labile phenomenon in many patients, varying in response to different stimuli, such as exercise or medication. Therefore, a patient can have a pronounced gradient during one study and none during the next. In addition, it must be stressed that systolic anterior motion of the mitral valve is a finding not restricted to HCM.

Some patients have a midventricular dynamic obstruction within the left ventricular cavity with a typical late peaking flow profile. The midcavity component might be missed unless the left ventricular cavity and its regional flow characteristics are examined carefully. 

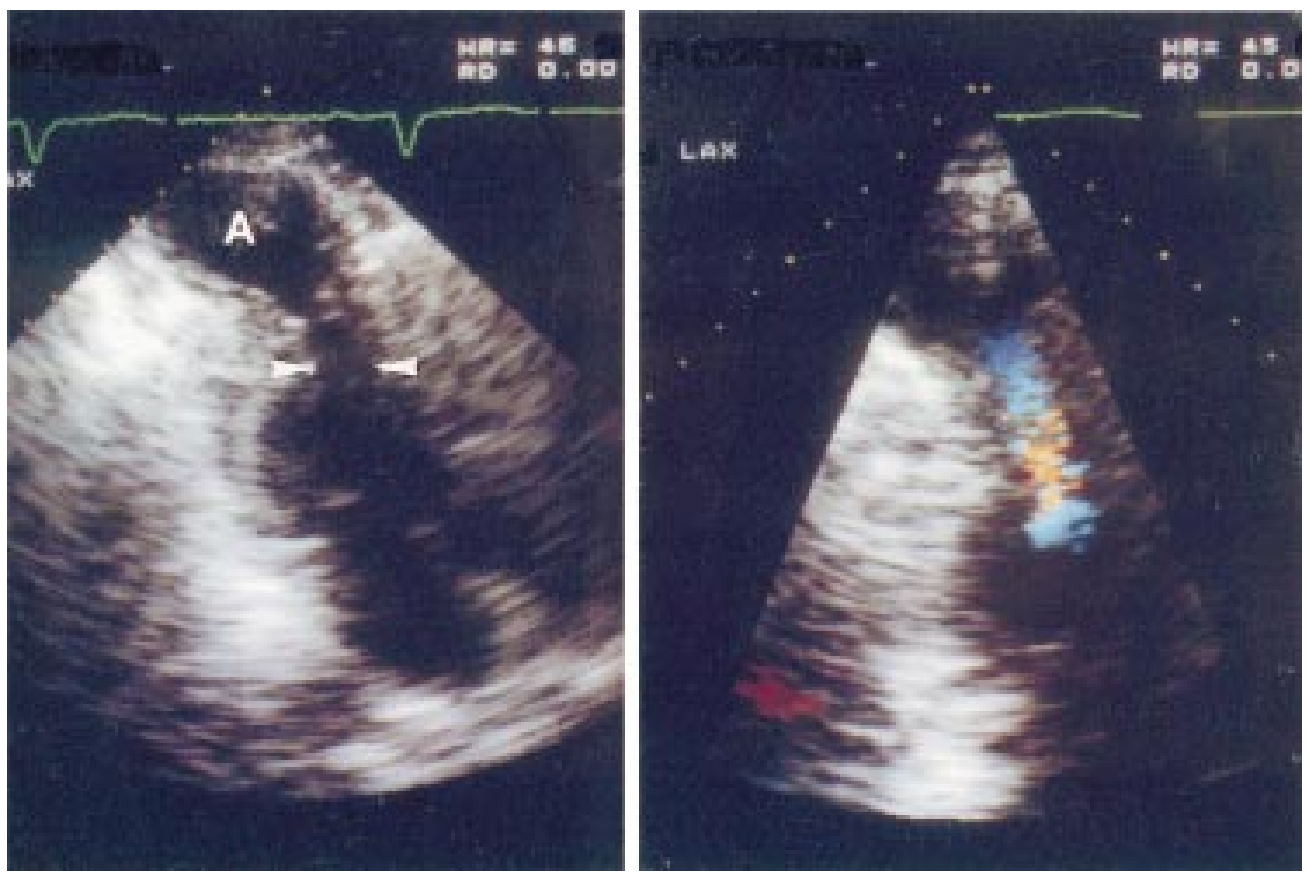

Figure 5 Apical long axis view with colour flow mapping in a patient with hypertrophic cardiomyopathy and a midventricular gradient and an apical aneurysm. Note, on the left, a midventricular narrowing of the left ventricular cavity (arrowheads) and, on the right, the turbulent flow on colour Doppler echocardiography originating at that level. A, apical aneurysm.

When the hypertrophy is localised or associated with a midcavity gradient there might be aneurysmal dilatation of the apex. Evidence of such "aneurysms" on echocardiography can lead to an erroneous diagnosis of ischaemic heart disease if care is not taken to assess regional wall thickening (fig 5).

\section{Pitfalls}

With such an emphasis placed on echocardiography for the diagnosis of HCM, a relatively rare condition with a wide phenotypic spectrum, it is not surprising that there is much potential for diagnostic error. Table 1 lists some of the problems commonly encountered during routine echocardiography of patients suspected of having HCM. These can occur singularly or in combination and lead to either incorrect diagnosis of HCM, or a missed diagnosis. Rigorous use of standard views with attention to detail such as adequate image quality, correct angulation and axis, and careful measurements should minimise errors. Importantly, in view of the serious individual consequences that the diagnosis of HCM may confer on the patient, it is crucial that the echocardiogram is interpreted in the clinical context by an expert, and that individual counselling is available.

\section{False positives}

If the echocardiographic hallmark for the diagnosis of HCM is ventricular hypertrophy, several conditions or anatomical variants may simulate LVH and can lead to misdiagnosis. Left ventricular false tendons and the presence of sigmoid septum, often seen in the elderly, are the most common causes of misdiagnosis of HCM. The presence of left ventricular false tendons or the moderator band of the right ventricle are regular echocardiographic find- ings of the normal heart that can give the impression of increased wall thickness when viewed from parasternal long axis projections. False tendons often manifest as linear echoes within the left ventricular cavity running parallel to the ventricular septum (fig 6). Pierard and colleagues ${ }^{21}$ reported prominent false tendons in nine of 300 consecutive patients referred for echocardiography that potentially could lead to diagnostic confusion. The most frequent site was from the septum to the lateral papillary muscle. Typically, they are long, thin structures that are hardly seen at all because they run parallel to the septum. Careful examination of the suspect area in different planes, particularly in the parasternal short axis projections, tracing the origins of the tendons, can usually provide the differential diagnosis.

The second most common misinterpretation for septal hypertrophy is the moderator band, which runs parallel to the septum in the right ventricle. This, together with inappropriate gain controls, can lead to unclear border delineation of the septal thickness and create septal hypertrophy. Again, careful investigation of the ventricular septum from short axis projections, together with appropriate gain settings, should ensure that results are not misinterpreted. The absence of increased wall thickness in other parts of the ventricle, a completely normal ECG, and absence of a family history of HCM are also important clues to the correct diagnosis.

The sigmoid septum has also been called the "septal bulge" or "angulated septum" and often leads to an erroneous diagnosis of asymmetric septal hypertrophy (fig 7). One of the most common interpretative errors of asymmetric septal hypertrophy is encountered in older patients, where the proximal portion of 

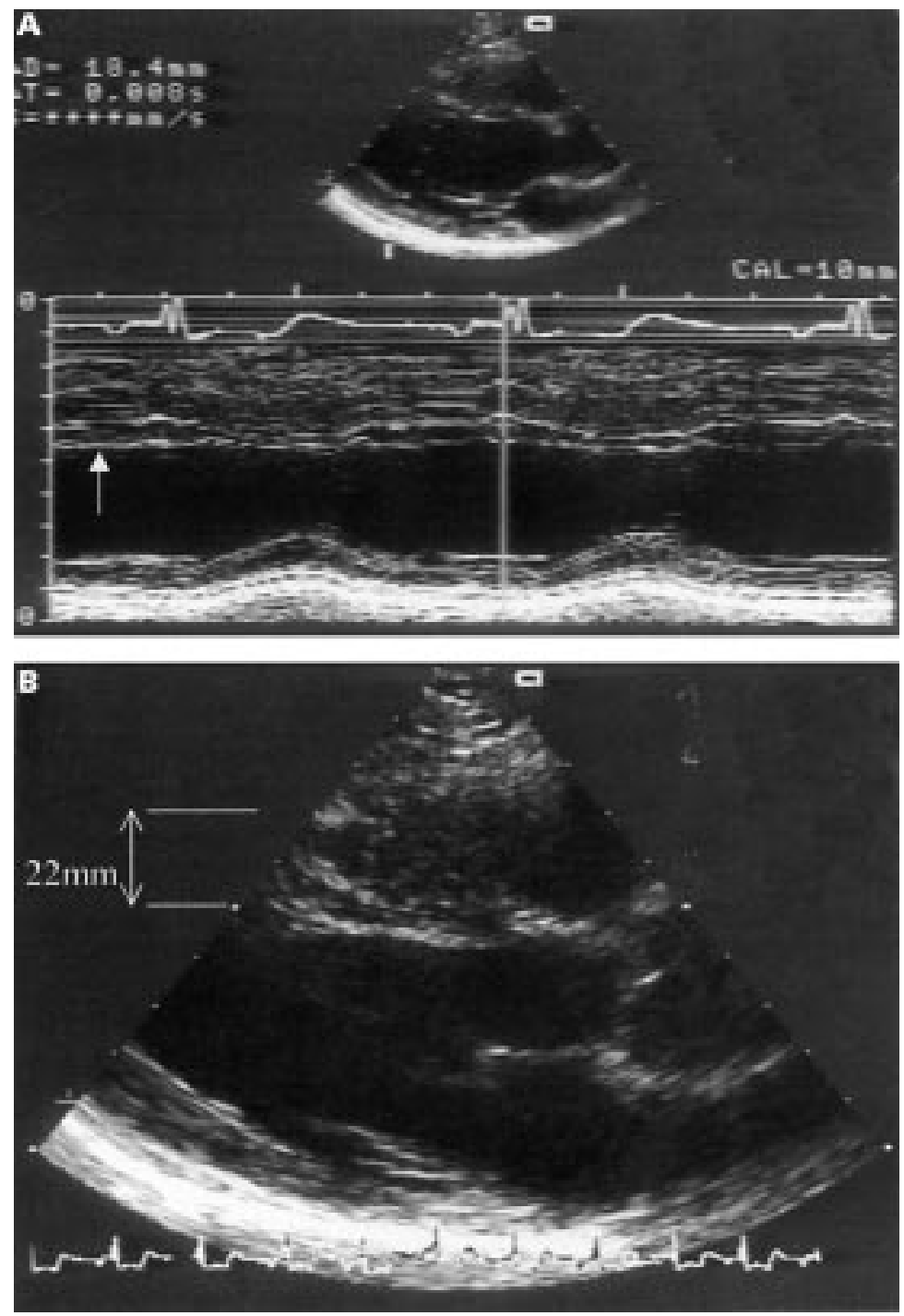

Figure 6 Parasternal long axis view from a patient referred with the echocardiographic diagnosis of hypertrophic cardiomyopathy (HCM). (A) The M mode image showing the normal septal thickness $(10 \mathrm{~mm})$. Notice that the measurement excludes the false tendon (arrow), which runs parallel to the septum. (B) The false tendon along the ventricular septum, which was included in the original measurement of $22 \mathrm{~mm}$ and led to the wrong diagnosis of HCM. can, however, be systolic anterior motion of the mitral valve and/or a left ventricular outflow tract gradient as a result of the shape of the left ventricular outflow tract, but this is not sufficient to confer the diagnosis of HCM. Increased, left ventricular wall thickness and left atrial size, which are often seen in the elderly, are usually thought of as a consequence of hypertension and/or increased ventricular stiffness, a "normal" aging process. Conversely, the presence of a positive family history for HCM should be suggestive of a particular genotype of HCM, associated with the myosin binding protein $\mathrm{C}$ mutations that often manifests in the elderly. ${ }^{24} 25$

Asymmetrical hypertrophy of the ventricular septum is not specific to HCM and can also occur in other clinical settings, such as systemic hypertension. ${ }^{26}$ Apparent asymmetric septal hypertrophy can also occur in conditions that cause thinning of the left ventricular posterior wall relative to the septum. This abnormal septal to posterior wall thickness ratio is commonly encountered in patients with coronary artery disease, either because of segmental hypertrophy of the septum (secondary to systemic hypertension) or, more commonly, as a result of transmural myocardial infarction with thinning of the posterior wall, thus leading to an abnormal septal to posterior wall ratio.

Finally, asymmetric septal hypertrophy is relatively common in infants with congenital heart disease. The overall prevalence of disproportionate septal hypertrophy in one study was $10 \%$, and in patients with pulmonary stenosis or pulmonary hypertension, this figure even exceeded $20 \%{ }^{27}$

Diagnostic difficulties frequently occur in athletes. The term "athlete's heart" is used to describe a constellation of features seen in elite athletes. Essentially, they are morphological adaptations to extreme physical training. There might be a mild increase in wall thickness, but this is rarely more than $16 \mathrm{~mm}$ in white athletes. Spirito and colleagues ${ }^{28}$ assessed 947 multidisciplinary national and international athletes and found that $<2 \%$ had a wall thickness similar to that found in "mild" HCM (in the range $12-16 \mathrm{~mm}$ ). Most of these were rowers or endurance cyclists with a large body surface area. In addition, athletes usually have a normal size left atrium and absence of family history of HCM. Perhaps the most distinctive feature of the athlete's heart, with or without increased wall thickness, is the increased ventricular chamber dimensions (end diastolic diameter $>55 \mathrm{~mm}$ ), in contrast to the generally small ventricular dimensions seen in HCM. If any doubt remains, a metabolic exercise test with a normal or supernormal maximum venous oxygen content and normal blood pressure response is unlikely to be found with pronounced cardiac pathology.

Rarely, infiltrative disorders such as amyloidosis may be confused with HCM. The reduced contraction of the amyloid left ventricle contrasts with the vigorous contraction usually seen in HCM. However, in the early stages of cardiac amyloidosis function might not be suppressed. Additional features, by echocardiography is unknown. Krasnow ${ }^{23}$ recently reported 94 such cases, noting that no other features of HCM were present. There 



Figure 7 Parasternal long axis view from a patient referred with the echocardiographic diagnosis of hypertrophic cardiomyopathy. (A) The $M$ mode image showing the original measurement of $20 \mathrm{~mm}$ for the ventricular septum passing obliquely through the angulated (sigmoid) septum. (B) The two dimensional picture with the angulated septum showing the correct measurement of the septum $(10 \mathrm{~mm})$.

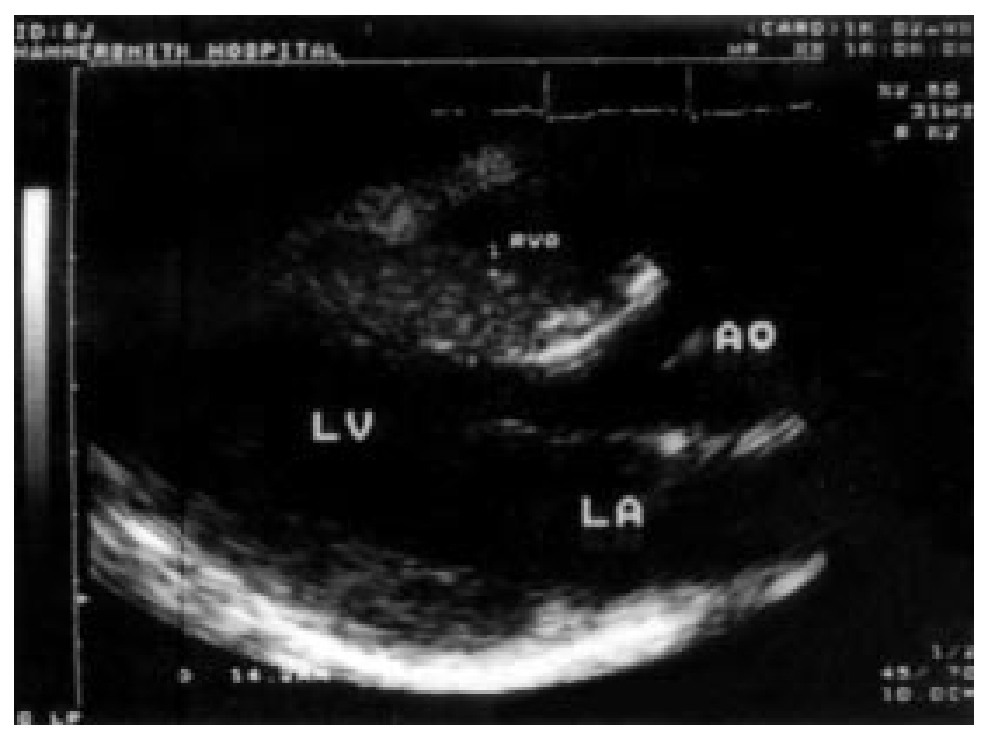

Figure 8 Parasternal long axis from a patient with Friedreich's ataxia demonstrating asymmetric septal hypertrophy. Reproduced with permission from Dutka et al. ${ }^{29}$ such as altered myocardial texture, presence of pericardial effusion and thickening of the valves and atrial septum, are often cited but all are subjective signs and none is specific on its own. A more simple and accurate means of differentiating amyloid from HCM is to examine the ECG, which is typically of low voltage in patients with amyloid, in contrast to the pronounced hypertrophy seen in HCM. Haemochromatosis in patients with frequent transfusions produces iron deposition within myofibres, which leads to ventricular dilatation, with concentric wall thickening and reduced compliance (restriction). Patients with Friedreich's ataxia, the most common of the spinocerebellar ataxias inherited as an autosomal recessive disorder, can present with a variable degree of ventricular hypertrophy. In one study of 55 patients with Friedreich's ataxia, ${ }^{29}$ we found left ventricular hypertrophy in 21 patients. The pattern of hypertrophy was variable, including asymmetric and concentric hypertrophy (fig 8).

The incidental detection of an intraventricular gradient with Doppler echocardiography can often be misleading and result in the misdiagnosis of HCM. Intraventricular gradients are not specific for the diagnosis of HCM and can occur in other conditions, with or without the ventricular hypertrophy. Small left ventricular cavity dimensions together with a vigorous left ventricular function, either as a result of a hyperdynamic state or hypovolaemia, can lead to a gradient. A typical example is in hypertensive patients on dialysis, who can mimic the picture of HCM.

\section{False negatives}

Although asymmetrical septal hypertrophy is the classic phenotype in HCM, it is important to appreciate that the hypertrophy might also be concentric, eccentric, or apical if the diagnosis is not to be missed. Distal or apical hypertrophy is only evident on sequential short axis examination of the left ventricle, and from the apical views, and can be easily overlooked. If the ECG shows deeply inverted $\mathrm{T}$ waves in the chest leads there should be a high degree of suspicion for apical HCM. ${ }^{30}$ However, a foreshortened apical four chamber view can give a false positive finding, with the appearance of increased distal wall thickness as a result of the oblique cut. Although distal hypertrophy is common in Japanese patients, it is also frequent in hypertensive patients, thus excluding the diagnosis of HCM as such, unless there is a positive family history of HCM.

Patients with mild concentric left ventricular hypertrophy present a diagnostic conundrum because this is the predominant pattern in secondary hypertrophy, such as hypertensive heart disease. Again, features favouring HCM are a positive family history, a small dynamic left ventricular cavity, characteristic ECG changes, normal 24 hour blood pressure, and an abnormal blood pressure response to exercise. A careful review of the echocardiogram might also reveal a more severe localised increase in wall thickness or involvement of the 
right ventricle, which point to a diagnosis of HCM. However, massive hypertrophy can be seen in neonates and children with glycogen storage disorders, but rarely in patients who do not have HCM. Studies differ in their definition of concentric hypertrophy because of differences in the morphological assessment of the segmental distribution of hypertrophy. In a recent study by Klues and colleagues ${ }^{7}$ of 600 patients, true concentric hypertrophy was rare in HCM, but about $30 \%$ had involvement of three or more contiguous regions of the left ventricle. Using conventional criteria this would have been classified as concentric hypertrophy.

\section{Children and adolescents}

Frequently in the context of family screening, one may be asked to examine a child or adolescent to look for ventricular hypertrophy. It has long been recognised that left ventricular hypertrophy is relatively unusual in childhood, but typically develops during the pubertal growth spurt. Additional information in the form of a detailed family history and ECG and, when possible, metabolic exercise stress testing, should be carefully sought. The ECG is more sensitive than the echocardiogram for the diagnosis of HCM in children, but less specific. Until recently, family screening has involved periodic echocardiographic evaluation of first degree relatives up to the age of 25 years. The recent recognition of non-penetrance, with phenotypically normal "carriers", and myosin binding protein $\mathrm{C}$ mutations with age related penetrance of $\mathrm{LVH}$, complicates the issue. In this context, a thorough family history, including the age of diagnosis of the affected individuals, is extremely important. A normal parent or normal echocardiographic evaluation at the age of 25 does not necessarily exclude the diagnosis, but careful pedigree analysis should identify gene carriers.

Hypertrophic cardiomyopathy is caused by a mutation in one of several sarcomeric proteins. Recently, it has become clear that some mutations, especially troponin $\mathrm{T}$ mutations, often manifest in only mild left ventricular hypertrophy (12-15 mm wall thickness), but have a high risk of sudden death. However, these are rare mutations occurring in approximately $15 \%$ of all patients with $\mathrm{HCM}{ }^{31}$ Strict adherence to previous criteria would not permit diagnosis of hypertrophic cardiomyopathy in these individuals. Therefore, care should be taken to assess the morphological findings in the context of the family history and some background knowledge of the mutations. The ECG is clearly abnormal in most cases. Figures 6 and 7 show examples of two such families. Importantly, the severity of LVH can vary greatly between individuals within the same family and, in general, it does not correlate with prognosis.

Some patients can present with a normal or mildly increased wall thickness, even though they have a highly malignant family history of sudden death. ${ }^{8}$ Others with an identified gene, and thus diagnosis of HCM, might not have any ventricular hypertrophy and only show some discrete ECG abnormalities. ${ }^{32}$ Left ventricular cavity enlargement is common in such patients. Differentiation from other causes of systolic dysfunction is necessary but can be very difficult. Previously documented hypertrophy is definitive but otherwise family history and a bizarre ECG, or if indicated, myocardial biopsy point to the correct diagnosis. In general, documented LVH in the absence of hypertension and a severe intramyocardial conduction abnormality in a patient with left ventricular systolic dysfunction should raise the possibility of HCM.

Explanted hearts from individuals with a pretransplant diagnosis of restrictive cardiomyopathy often reveal extensive myofibrillar disarray in the absence of increased wall thickness. Therefore, it is important to be aware of the possibility of HCM in patients presenting with a restrictive physiology. Echocardiographic evaluation of family members might reveal individuals with the same phenotype or that of a more typical HCM.

\section{The way forward}

It is clear that although echocardiography is an invaluable tool in the diagnosis and follow up of patients with HCM it is not infallible. Totally reliable diagnostic accuracy can only be achieved with genotyping, which is not yet possible for all subgroups. Newer echocardiographic technologies looking at myocardial velocities and gradients might be helpful. Until then, an interractive diagnostic protocol with a complete, standardised echocardiogram is the best we can offer.

1 Richardson P, McKenna W, Bristow M, et al. Report of the 1995 World Health Organisation/International Society and Federation of Cardiology task force on the definition and classification of cardiomyopathies. Circulation 1996;93:841-2.

2 Maron BJ, Bonow RO, Cannon RO, et al. Hypertrophic cardiomyopathy; interrelation of clinical manifestations, pathophysiology, and therapy. N Engl f Med 1987;316:780pathophysiol $844-52$.

3 Wigle ED, Sasson Z, Henderson MA, et al. Hypertrophic cardiomyopathy: importance of the site and extent of hypertrophy - a review. Prog Cardiovasc Dis 1985;28:1-83.

4 Shapiro LM, McKenna WJ. Distribution of left ventricular hypertrophy in hypertrophic cardiomyopathy: a two dimensional echocardiographic study. $7 \mathrm{Am}$ Coll Cardiol 1983;2:437-44

5 Maron BJ, Nichols PF, Pickle LW, et al. Patterns of inheritance in hypertrophic cardiomyopathy: assessment by Cardiol 1984;53:1087-94

6 Maron BJ, Gottdiener JS, Epstein SE. Patterns and significance of distribution of left ventricular hypertrophy hypertrophic cardiomyopathy: a wide-angle Am 7 Cardiol 1981;48:418-28

7 Klues HG, Schiffers A, Maron BJ. Phenotypic spectrum and patterns of left ventricular hypertrophy in hypertrophic cardiomyopathy: morphologic observations and significardiomyopathy: morphologic observations and signifi600 patients. F Am Coll Cardiol 1995;26:1699-708.

8 McKenna WJ, Stewart JT, Nihoyannopoulos P, et al. Hypertrophic cardiomyopathy without hypertrophy: two families with myocardial disarray in the absence of increased myocardial mass. Br Heart f 1990;63:287-90.

9 McKenna WJ, Spirito P, Desnos M, et al. Experience from clinical genetics in hypertrophic cardiomyopathy: proposal for new diagnostic criteria in adult members of affected families. Heart 1997;78:130-4.

10 Nihoyannopoulos P, McKenna WJ. Hypertrophic cardiomyopathy. In: Roelandt JRTC, Sutherland GR, Iliceto S, et al, eds. Cardiac ultrasound. Edinburgh: Churchill Livingstone, 1993:371-89.

11 Brenner JI, Baker K, Ringel RE, et al. Echocardiographic evidence of left ventricular bands in infants and children. $\mathcal{F}$ Am Coll Cardiol ventricular bands in

12 Goor D, Lillehei CW, Edwards JE. The sigmoid septum; variation in the contour of the left ventricular outlet. $A m \mathcal{f}$ Roentgenol Radium Ther Nucl Med 1969;107:366-76. 
13 Hanrah P, Mathey DG, Siegert R, et al. Left ventricular relaxation and filling pattern in different forms of left ventricular hypertrophy: a
Cardiol 1980;45:15-23.

14 Sanderson JE, Trail TA, St John Sutton MG, et al. Left ventricular relaxation and filling in hypertrophic cardiomyopathy: an echocardiographic study. Br Heart $\mathcal{f}$ 1978;40:596-601.

15 St John Sutton MG, Tajik AJ, Gibson DG, et al. Echocardiographic assessment of left ventricular filling and septal and posterior wall dynamics in idiopathic hypertrophic subaortic stenosis. Circulation 1978;57:512-20.

16 Doi YL, McKenna WJ, Gehrke J, et al. M mode echocardiography in hypertrophic cardiomyopathy: diagnostic criteria
and prediction of obstruction. Am $\mathcal{F}$ Cardiol 1980;45:6-14.

17 Appleton CP, Hatle LK, Popp RL. Relation of transmitral flow velocity patterns to left ventricular function: new insights from a combined hemodynamic and Doppler echocardiographic study. F Am Coll Cardiol 1988;12:42640 .

18 Maron BJ, Spirito P, Green KJ, et al. Non-invasive assessment of left ventricular diastolic function by pulsed Doppler echocardiography in patients with hypertrophic

19 Nihoyannopoulos P, Karatasakis G, Frenneaux M, et al. Diastolic function in hypertrophic cardiomyopathy; relation to exercise capacity. $\mathcal{F}$ Am Coll Cardiol 1992;19:53640.

20 Takenaka K, Dabestani A, Gardin JM, et al. Left ventricular filling in hypertrophic cardiomyopathy: a pulsed Doppler echocardiographic study. 7 Am Coll Cardiol 1986;7:126371 .

21 Pierard LA, Henrard L, Noel JF. Detection of left ventricular false tendons by two-dimensional echocardiography. Acta Cardiol 1985;40:229-35.

22 Goor D, Lillehei CW, Edwards JE. The "sigmoid septum" Variation in the contour of the left ventricular outflow tract. Am f Roentgenol Radium Ther Nucl Med 1969;107:366-76.
23 Krasnow N. Subaortic septal bulge simulates hypertrophic cardiomyopathy by angulation of the septum with age, independent of focal hypertrophy. An echocardi study. F Am Soc Echocardiogr 1997;10.545-55.

24 Bonne G, Carrier L, Bercovici J, et al. Cardiac myosin binding protein-C gene splice acceptor site mutation is associated with familial hypertrophic cardiomyopathy. Nat Genet 1995;11:438-40.

25 Watkins H, Conner D, Thierfelder L, et al. Mutations in the cardiac myosin binding protein-C on chromosome 11 cause familial hypertrophic cardiomyopathy. Nat Genet 1995;11:434-7.

26 Lewis JF, Maron BJ. Diversity of patterns of hypertrophy in patients with systemic hypertension and marked left ventricular wall thickening. Am f Cardiol 1990;65:874-81.

27 Maron BJ, Clark CE, Henry WL, et al. Prevalence and characteristics of dysproportionate ventricular septal thickening in patients with acquired or congenital heart disease: echocardiographic and morphologic findings. Circulation 1977;55:489.

28 Spirito P, Pelicia A, Proschan MA, et al. Morphology of "athlete's heart" assessed by echocardiography in 947 elite athlete's heart" assessed by echocardiography in 947 elite 6 .

29 Dutka DP, Donnelly JE, Nihoyannopoulos P, et al. Marked variation in the cardiomyopathy associated with Friedreich's ataxia. Heart 1999;81:141-7.

30 Alfonso F, Nihoyannopoulos P, Stewart J, et al. Clinical significance of giant T-waves in hypertrophic cardiomyopathy. f Am Coll Cardiol 1990;15:965-71.

31 Moolman JC, Corfield VA, Posen B, et al. Sudden death due to troponin T mutations. f Am Coll Cardiol 1997;29:54955.

32 Charron $\mathrm{Ph}$, Dubourg O, Desnos $\mathrm{M}$, et al. Familial hypertrophic cardiomyopathy. Diagnostic value of electrocardiography and echocardiography for familial hypertrophic cardiomyopathy in a genotyped adult population. Circulation 1997;96:214-19. 